ARTICLE

Received 9 Dec 2013 | Accepted 14 Feb 2014 | Published 28 Mar $2014 \quad$ DOl: 10.1038/ncomms4456

\title{
Multimodal label-free detection and discrimination for small molecules using a nanoporous resonator
}

Jinsung Park ${ }^{1, \star}$, Doyeon Bang ${ }^{2,3, \star}$, Kuewhan Jang ${ }^{4, \star}$, Eunkyoung Kim ${ }^{2,3}$, Seungjoo Haam ${ }^{2} \&$ Sungsoo $\mathrm{Na}^{4}$

To detect chemical or biological threats, it is crucial that sensor devices can differentiate various target molecules. In general, each different sensing method has its own strengths and weaknesses due to their respective limitations. For example, although resonant sensors have high sensitivity, they are not able to discriminate target molecules. At the same time, although surface-enhanced Raman spectroscopy is a representative label-free detection method that can discriminate target molecules, its fabrication is often complex and expensive. Here we present a label-free multimodal nanoporous resonator-based system for small molecule detection and discrimination that combines the strengths of each of these sensing methods. Our approach is not only able to improve the sensitivity of the resonant sensor but it can also discriminate the target molecules. Furthermore, the fabrication process is swift (lasting $<3 \mathrm{~min}$ ) and convenient.

\footnotetext{
${ }^{1}$ Department of Control and Instrumentation Engineering, Korea University, Jochiwon 339-700, Republic of Korea. ${ }^{2}$ Department of Chemical and Biomolecular Engineering, Yonsei University, Seoul 120-749, Republic of Korea. ${ }^{3}$ Active Polymer Center for Pattern Integration (APCPI), Yonsei University, Seoul 120-749, Republic of Korea. ${ }^{4}$ Department of Mechanical Engineering, Korea University, Seoul 136-701, Republic of Korea. ${ }^{\star}$ These authors contributed equally to this work. Correspondence and requests for materials should be addressed to S.H. (email: haam@yonsei.ac.kr) or to S.N. (email: nass@korea.ac.kr).
} 
O ver the past two decades, the field of sensing has advanced to include a wide range of methods and devices for chemical, biological and environmental toxicity detection $^{1}$. The monitoring of chemical and biological targets is important for medical diagnostics and bio-warfare applications as well as for fundamental research ${ }^{2}$. In addition, because of the remarkable developments that have taken place recently in the field of nanoscale research and industry, the detection range for nanotoxic materials that can adversely affect the environment and human health has been greatly increased ${ }^{3}$. Common methodbased molecular sensing involves labelling processes such as quantum dots, fluorescent markers and dyes ${ }^{4}$. Although labelled detection is widely used in molecular sensing, photochemical degradation, $\mathrm{pH}$-dependence, time constraints and high costs are all important considerations that can restrict the use of these methods ${ }^{2,5}$. For the various target scopes and overcoming the limitation of labelling method, current developing techniques are focused on maximizing sensitivity, label-free detection, reducing the cost and creating multimodal systems for analytical detection of target molecules ${ }^{6}$.

Resonant sensors are highly sensitive devices that provide a method for the label-free quantitative measurement of molecular interactions ${ }^{7,8}$. The resonant sensor is one of the cantilever-based detection modes, where the binding of the molecule to the cantilever surface changes the resonant frequency of the cantilever. Thus, the resonant sensor detects the surface molecular binding directly, as the resonant frequency is a function of the cantilever mass. From this background, the resonant sensor has shown the highest potential for achieving the most sensitive measurements of molecular interactions such as enzyme-peptide interactions $s^{9,10}$, nucleotide interactions ${ }^{11}$ and protein-antibody interactions ${ }^{12,13}$. Recently, we have shown that a resonant sensor was able to detect the nanotoxic material $\left(\mathrm{Ag}^{+}\right)$in the real environment ${ }^{14}$. While the resonant sensing platform has high sensitivity and economic benefit due to the label-free method, the discrimination of binding molecules and time-consuming tasks for exploring in different configuration to enhance the sensitivity (using MEMS and NEMS technology) are major drawback of the resonant sensor ${ }^{8,13}$.

Another well-established label-free detection method capable of single-molecule detection is surface-enhanced Raman spectroscopy (SERS). SERS is a phenomenon in which the intensity of light scattered from the molecule is amplified when light is shone on the molecules placed on nanostructured metallic surface ${ }^{15}$. SERS has drawn great attention as an alternative method for small molecule detection due to its multiplexing capability since the intensities and and frequencies of vibrational transitions measured in Raman spectra provide unique finger-printing spectra of molecular species ${ }^{16}$. Various strategies have been reported for fabricating nanostructured metallic platforms for efficient use as SERS substrates ${ }^{17-19}$. However, these nanostructured SERS substrates have several drawbacks such as a complex fabrication process, lack of uniformity at large scale and MEMS incompatibility.

To overcome the above problems related to resonant sensor and SERS, we propose a novel approach for discriminating binding molecules and improving the sensitivity of resonant sensors at detection limits of $10^{-9} \mathrm{M}$ without amplification by making use of nanoporous sensors, in particular, multimodal nanoporous resonators (MNPR). As a result of their large surface area, nanoporous structures have been widely used for drug delivery $^{20}$, water filtration ${ }^{21,22}$, batteries ${ }^{23}$ and various sensor applications ${ }^{24-27}$. Herein, we adopt MNPR for small molecules detection such as $p$-aminothiophenol ( $p$-ATP) and Malachite green isothiocyanate (MG), which are representative small molecules for Raman spectroscopy to verify the SERS ${ }^{28-31}$.
Moreover, the molecular weight of p-ATP (125.19 Da) is light enough to ensure the high sensitivity of MNPR by comparison with previous studies ${ }^{9-1}$. It is remarkably shown that MNPR enables one to label-free detect and discriminate small molecules, not only $\sim 10,000$ times improved sensitivity compared with commercialized resonator but also discrimination of binding molecule individually. In addition, the fabrication of MNPR is a swift (within $3 \mathrm{~min}$ ) and convenient process that leads to reducing the cost.

\section{Results}

MNPR frabrication. An MNPR for the label-free detection and discrimination of small molecules was designed by modifying a commercialized micro-resonator using an electrochemical route as shown in Fig. 1 (see Supplementary Fig. 1). The fabrication process for creating the MNPR involved two convenient steps. The first step was to induce $\mathrm{Au} / \mathrm{Ag}$ alloy growth on a bare gold surface. Generally, the surface of a micro-cantilever is coated with a material such as gold $(\mathrm{Au})$, chrome $(\mathrm{Cr})$ or aluminium $(\mathrm{Al})$ in order to enhance laser reflection, which will ensure a clear resonance peak and allow for high-precision atomic force microscopy (AFM) images. Au/Ag alloy was directly deposited onto the gold surface using a mixture of gold and silver-plating stock solutions over $3 \mathrm{~min}$. The second step was the selective wet etching of Ag using nitric acid $\left(\mathrm{HNO}_{3}\right)$, which took $3 \mathrm{~s}$. After gently washing process, the MNPR was ready to detect the small molecules (see Methods). These fabrication processes of the MNPR is facile to prepare comparing with other techniques. General micro/nanoporous sensors adopt the complicated procedures that include wet etching, polishing, micro/nano patterning, and so $\mathrm{on}^{32}$. In addition, similar de-alloying method for porous strucutre includes thermally evaporation with high vacuum system and additional enlarging the pore size process ${ }^{26}$. However, the fabrication process of the MNPR excludes expensive and timeconsuming processes as mentioned above. Target-specific binding generally refers to antibody- or aptamer-based sensing. The binding between thiolated small molecules and a gold surface is directly detected and discriminated as means of showing proof of concept. The Au/Ag alloy deposition and the MNPR were both measured using energy-dispersive X-ray spectroscopy (EDS), see Supplementary Fig. 2.

Multimodal detection strategy. The proposed multimodal sensing systems incorporate two detection methods. The first method is based on the shift in the resonant frequency of the cantilever upon the change of mass, which occurs when the molecule adheres to the surface. From previous studies, we have determined that the rectangular-shaped resonator exhibits resonant behaviour that is consistent with the classical elastic continuum model ${ }^{14,33}$. It is well known that mass change is a dominant factor in the resonant frequency shift of a thick micro-resonator as a result of the related molecular interactions such as molecular binding and/or molecular cleavage ${ }^{9,14}$. It means that the high sensitivity of resonance sensor is proportional to the mass increase and/or the probability of molecular adsorption. Here the sensitivity represents a general intensitiy of sensor response that is widely used for previous studies $^{10,34,35}$. A typical mass increasing method is that of a sandwich assay that involves an attachment of a subordinate amplifier to the target molecule ${ }^{36}$. However, the sandwich assay is not capable of quantitatively determining the number of target molecules. In addition for small molecular sensing, investigation of adjust amplifier is difficult due to the small number of binding sites available. Thus, the increase of probability for molecular adsorption is an appropriate alternative using nanoporous 

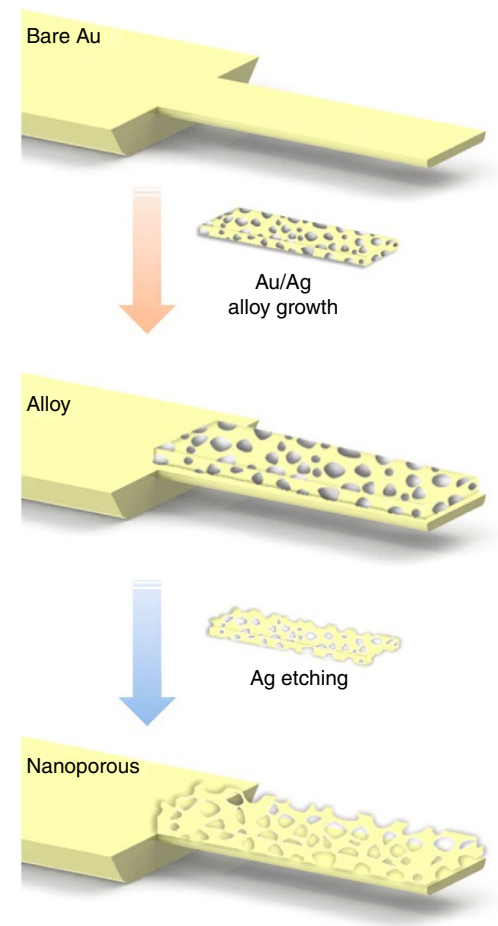
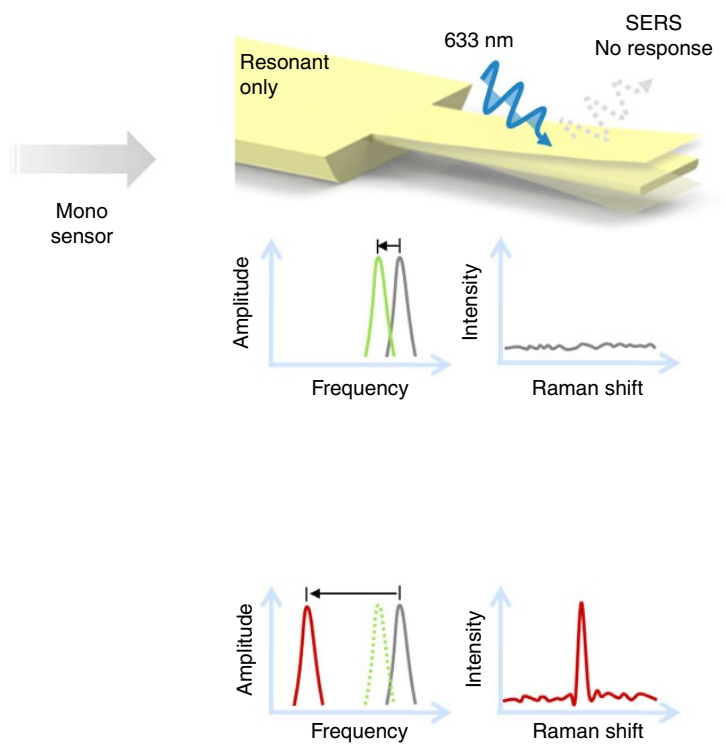

Multimodal sensor

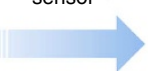

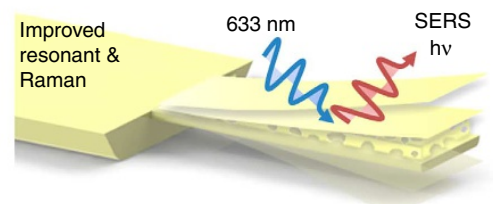

Figure 1 | Illustration of the fabrication procedures for the MNPR. An MNPR for the label-free detection and discrimination of small molecules was designed by modifying a commercialized micro-resonator. The modification involved two convenient steps. The first step was to induce Au/Ag alloy growth on a bare gold surface. Then, the selective wet etching of Ag using nitric acid produces a porous gold layer on the micro-resonator.

structures in MNPR. It means that the MNPR has better sensitivity than the commercialized resonant sensor due to the larger surface area. The second detection method in this work is based on SERS substrates. Recently, the development of nanostructured-metal-based SERS substrates has been of great interest since the magnitude of Raman cross-section can be greatly enhanced when the Raman-active molecules are placed around a nanostructured-metal surface ${ }^{37}$. It is attributed to plasmonic coupling between local nanostructured-metal in close proximity, which results in huge local electromagnetic field enhancements ${ }^{38}$. The location of spots of giant electromagnetic field enhancement is referred to as 'plasmonic hotspots 39 . Our MNPR is able to response the SERS due to lots of plasmonic hotspots and well-distributed plasmonic hotspots, which are dependent on the shape of nanostructure. Further, high-cost and complicated fabrication processes are not necessary for the MNPR manufacturing. Moreover, the target molecules were discriminated using SERS spectra, which show unique profiles for each molecule. From these standpoints for high sensitivity and target molecule discrimination, though bare resonator was able to detect the small molecules ( $p$-ATP) using a resonant frequency shift only, the bare resonator showed a deficiency for both points. In contrast, nanoporous structure of MNPR led to an improved resonant frequency shift and response of SERS. Further, the MNPR solves the shortcoming of each technique. In the viewpoint of the resonant sensor, unrecognized bound molecules are the critical drawback. Furthermore, SERS also have the drawback in which the sensitivity is dependent on the shape of the nanostructure. Consequently, our sensing platform complements to each technique using discrimination of target molecules and enhancement of sensitivity.

MNPR optimization. In order to find the optimal conditions for small-molecule detection, the surface morphologies and
Raman-mapped images of MNPRs were obtained using tappingmode AFM (tmAFM) and confocal Raman microscopy on the Au-containing plating stock solutions, respectively. Figure 2a showing the surface morphologies of MNPRs in the threedimensional (3D) images are rendered in red, green and violet colours, which indicate the deep (approximately $-180 \mathrm{~nm}$ ), flat $(0 \mathrm{~nm})$ and towering $(\sim 110 \mathrm{~nm})$ areas, respectively. As mentioned above, in the point of view for resonant sensor, high sensitivity is proportional to a large surface area since a larger surface area corresponds to an increase in the molecular adsorption probability. From the tmAFM 3D images, it is noticed that the decreased content of $\mathrm{Au}$ led to an increase in the surface area until the solution reached $12.5 \%(1 / 8)$ of $\mathrm{Au} / \mathrm{Ag}$ plating stock solution because vacancy among gold foams is increased. The surface area of lowest $\mathrm{Au}$ content (6.25\%) was only slightly decreased because the number of vacancies was sufficiently large to produce what could be considered a dead volume. In order to compare the surface areas definitively we employed a normalized surface area for Au content (Fig. 2b). The size of all tmAFM 3D images was $3 \times 3 \mu \mathrm{m}$, which was the standard area for normalization. Here the normalized surface area was described as $A_{\mathrm{n}}=A_{\mathrm{m}} / A_{\mathrm{b}}$, where $A_{\mathrm{m}}$ and $A_{\mathrm{b}}$ are the surface area of MNPR and bare resonator, respectively. As shown the Fig. 2b, the optimal Au content is $12.5 \%$ in stock solution, which gave a normalized surface area of $1.532 \pm 0.242$ (blue bar, $A_{n} \pm$ s.d.). To increase the statistical validity, normalized surface area of 10 different MNPRs were measured for each $\mathrm{Au}$ content. In parallel to the surface area analysis of MNPRs using AFM analysis, estimation of electrochemically active surface area was additionally investigated using cyclic voltammetric analysis of gold oxide formation and stripping (Supplementary Fig. 3). Significant anodic current takes place at a potential above $\sim 1.7 \mathrm{~V}$ due to surface oxide formation. Thereafter, the electric charge transferred during the reduction of gold oxide (reduction peak $\sim 0.7 \mathrm{~V}$ ) was used to estimate the 
a
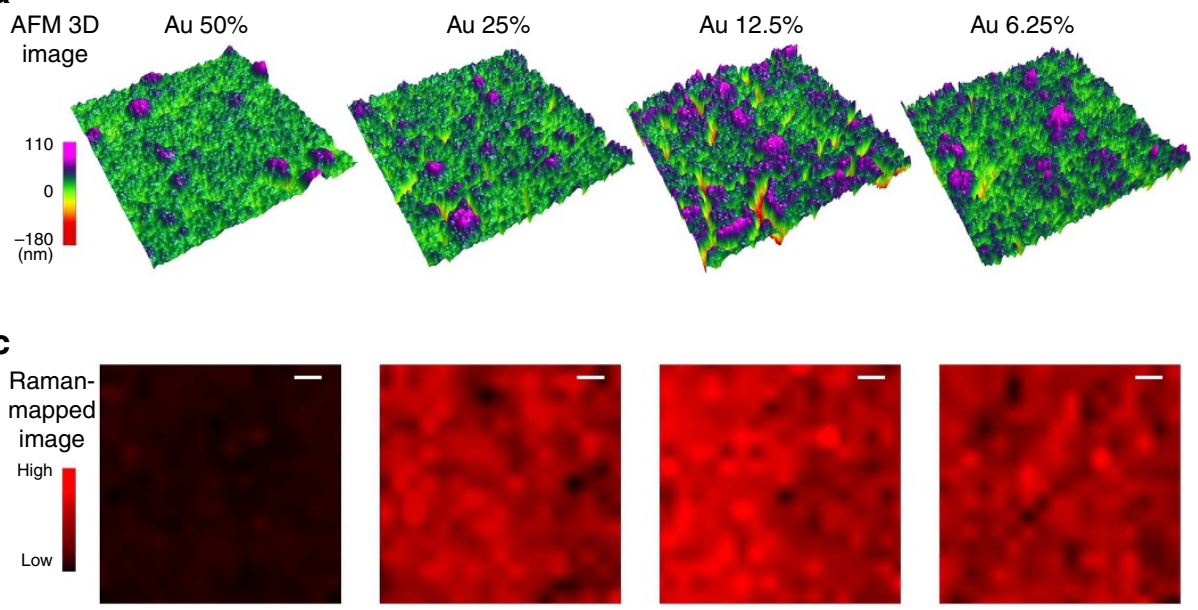

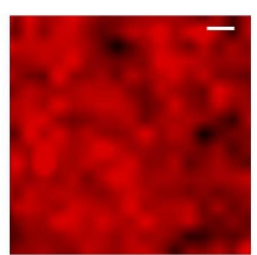

b

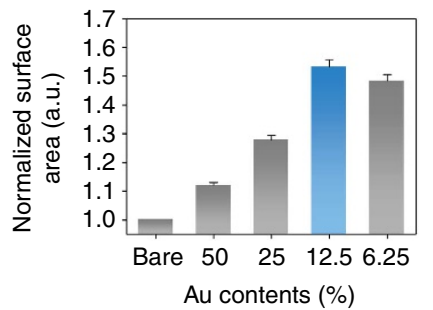

d

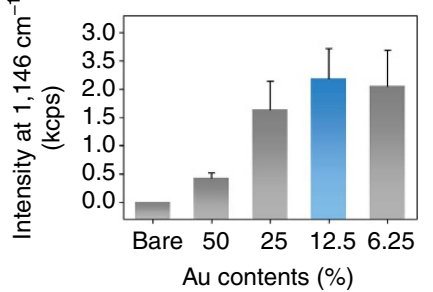

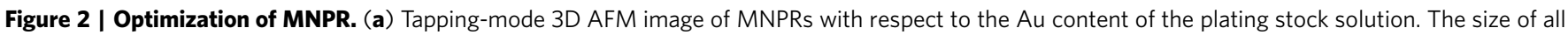
images was $3 \times 3 \mu \mathrm{m}$. (b) Normalized surface area with respect to the Au contents. For the statistical data, normalized surface areas of 10 different

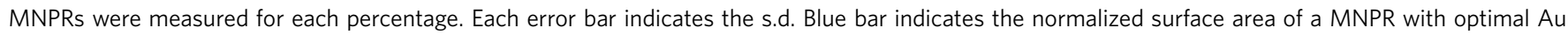
content (12.5\%). (c) Confocal Raman mapped images of MNPRs with respect to the Au content after deposition of 10 - 4 M of $p$-ATP molecules (scale bar $1 \mu \mathrm{m}$ ). The Raman signal was imaged between 1,140 and $1,145 \mathrm{~cm}^{-1}$. (d) SERS intensity with respect to the Au contents using $p$-ATP molecules at

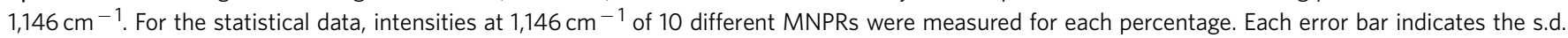
Blue bar indicates the SERS intensity of a MNPR with optimal Au content (12.5\%).

electrochemically active surface area. From these results, $12.5 \%$ of $\mathrm{Au}$ content was chosen for use in the MNPR fabrication. Even though the normalized surface area is not able to present an absolute value for MNPR from the tmAFM imaging method, the tendency of the surface area was able to be observed using tmAFM analysis. In order to determine the optimal conditions for SERS response, confocal Raman-mapped images were also observed with respect to Au content, which were widely used for evaluation of the SERS enhancement uniformity (Fig. 2c $)^{40}$. In order to evaluate the enhancement of the Raman scattering intensity for each sample, $p$-ATP $(100 \mu \mathrm{M})$ was deposited on each sample and the intensity of the Raman signal between 1,140 and $1,145 \mathrm{~cm}^{-1}$ that was mapped. It is noticable that the peak c.a. $1,142 \mathrm{~cm}^{-1}$ existed with considerable enhancement by $-\mathrm{N}=\mathrm{N}$ - stratching mode (so called ' $\mathrm{b}_{2}$ mode') of 4,4 ' dimercaptoazobenzene (DMAB), which was oxidatevly transformed from $p$-ATP during SERS measurement ${ }^{28,41,42}$. Raman-mapped images of MNPRs display that the Raman signal of highest $\mathrm{Au}$ content (50\%) show relatively weaker signal enhancement than samples with lower Au content due to the lack of plasmonic hotspots. Moreover, although the nanostructure of MNPRs was randomly organized, the enhancement of the Raman scattering was almost evenly distributed because the diameter of the illuminating laser beam $(1 \mu \mathrm{m})$ was sufficiently large to equalize the local structures. Figure $2 \mathrm{~d}$ shows the peak intensities of SERS from each MNPR. Further, for the statistical validity we measured the intensities at $1,146 \mathrm{~cm}^{-1}$ of 10 different MNPRs of each $\mathrm{Au}$ percentage. The optimal Au content utilized for the MNPRs as SERS substrates is $12.5 \%$ of Au content as seen from the confocal Raman-mapped images.

MNPR characterization. In order to investigate the characteristic properties of the MNPRs, scanning electron microscopy (SEM), tmAFM and Raman spectroscopy are used to determine their morphological properties, resonance behaviour and SERS responses (Fig. 3). Figure $3 a-c$ shows SEM images of the bare $\mathrm{Au}$ surface, the $\mathrm{Au} / \mathrm{Ag}$ alloy deposition surface and the MNPR surface, respectively. The scale bars of top, right and left indicate $100 \mu \mathrm{m}, 1 \mu \mathrm{m}$ and $100 \mathrm{~nm}$, respectively. Flat surface morphology was observed in the commercialized resonator (Fig. 3a). After the Au/Ag alloy deposition was complete, the surface roughness was seen to have increased relative to the bare Au surface (Fig. 3b). Here the ratio of Au content in Au and Ag plating stock solutions was $12.5 \%$, which was optimal for small molecule sensing as previously discussed. Ultimately, the nanoporous $\mathrm{Au}$ substrate was observed after the Ag-etching step was performed using nitric acid (Fig. 3c). The nanoporous structures were described as physically having two parts: deep trenches and small holes, which were the space for dissolved Ag. The width of deep trenches and small holes were $100-500 \mathrm{~nm}$ and $<100 \mathrm{~nm}$, respectively. For the depth measurement, we have employed the tmAFM images and profile for the MNPR surface (Fig. 3d). From the height profile, the average depth of the deep trenches and small holes was $\sim 135 \mathrm{~nm}$ and $\sim 30 \mathrm{~nm}$, respectively ( $>20$ profiles measurements). Herein, height profiles were only able to show the trend for the depth due to the limitation imposed by the radius of the tip of the microcantilever. The resonance behaviours of the bare Au state (grey line), $\mathrm{Au} / \mathrm{Ag}$ alloy deposition state (orange line) and MNPR (blue line) are shown in Fig. 3e. All these resonance peaks were determined using the same commercial cantilever using the fabrication protocol. It is shown that a decrease in the resonance frequency indicates an increase of mass relative to the bare $\mathrm{Au}$ surface, which is due to the $\mathrm{Au} / \mathrm{Ag}$ alloy deposition ${ }^{33}$. In contrast, the resonance peak increases as a result of the Ag etching, which is indicative of the related mass decrease. However, the resonance peak of the MNPR was still lower than that of the bare cantilever because of the $\mathrm{Au}$ nanoporous structures that remained. The final characteristic property of the MNPR measured was the SERS response of each state after deposition of $p$-ATP molecules $(100 \mu \mathrm{M})$ (Fig. 3f). For the case of the bare Au state or the Au/Ag alloy deposition state, the Raman spectrum is not observed because the quantity of the absorbed $p$-ATP on the sample substrate is too small to be observed using normal Raman scattering techniques. However, in case of the MNPR, the 

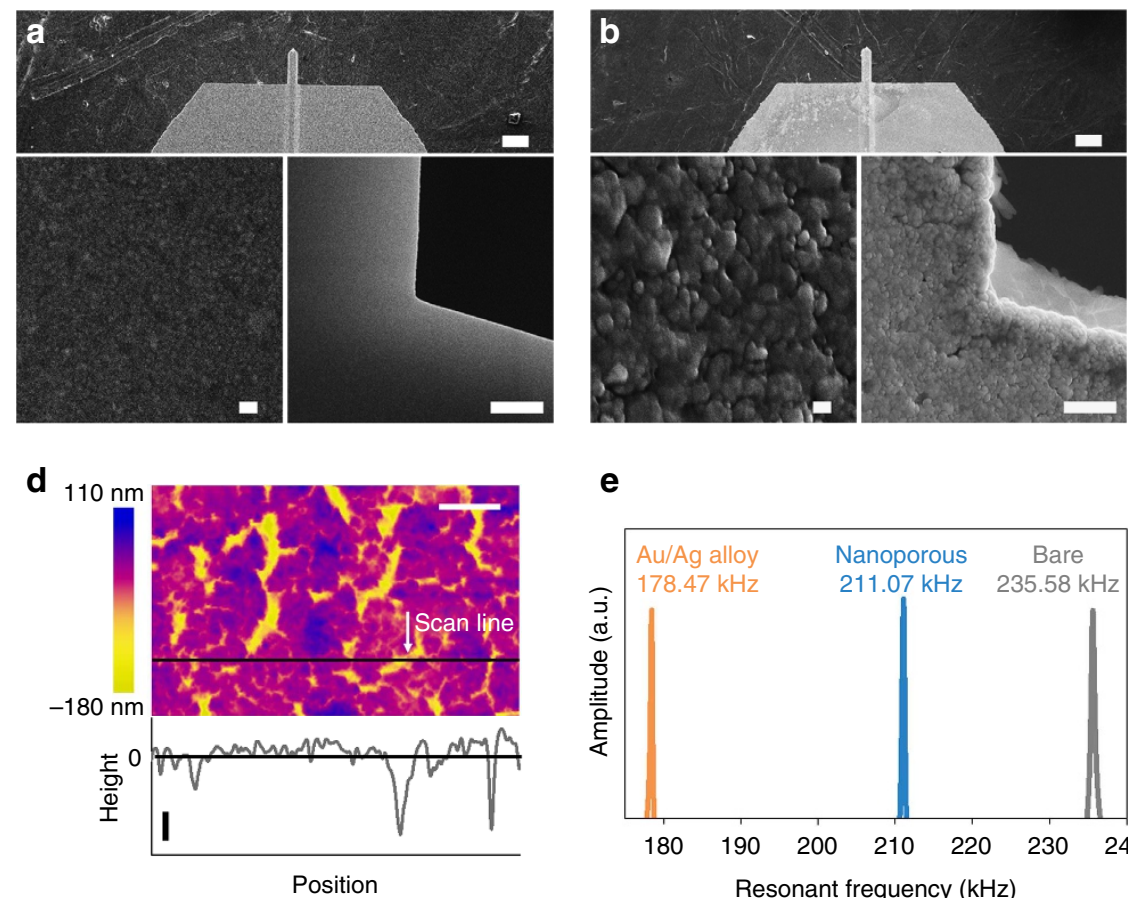

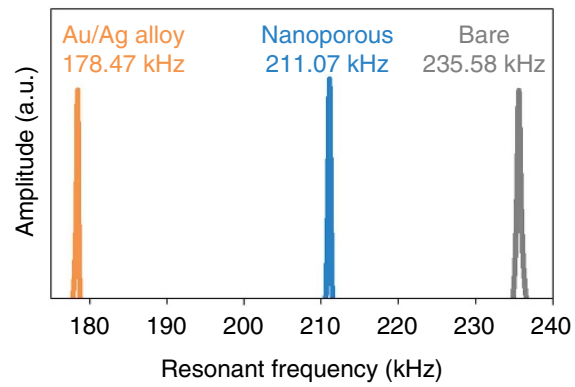

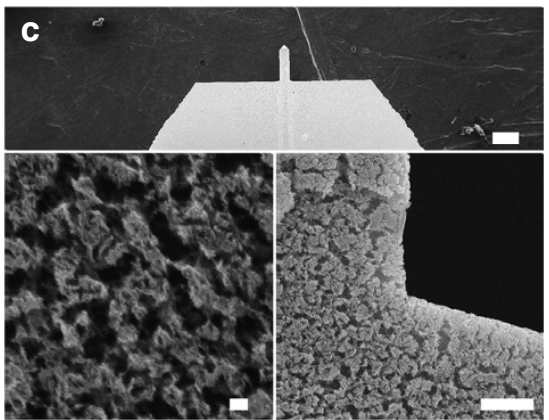

f

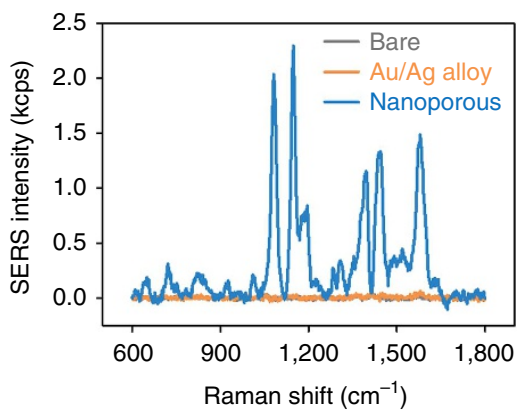

Figure 3 | Characterization of MNPR. SEM image of each resonator surface: (a) bare Au surface, (b) Au and Ag alloy surface, and (c) nanoporous surface (in each panel, top scale bar is $100 \mu \mathrm{m}$, bottom right scale bar is $1 \mu \mathrm{m}$ and bottom left scale bar is $100 \mathrm{~nm}$ ). (d) tmAFM image of nanoporous surface and its corresponding height profile (horizontal scale bar is $500 \mathrm{~nm}$ and vertical scale bar is $500 \mathrm{~nm}$ ). (e) Resonant frequency of bare Au, Au/Ag alloy, and nanoporous state of resonator. (f) SERS response of bare $\mathrm{Au}, \mathrm{Au} / \mathrm{Ag}$ alloy, and nanoporous state of resonator using $10^{-4} \mathrm{M}$ of $p$-ATP molecules.

Raman spectrum is observed due to the enhancement of the Raman scattering.

Resonant sensing. To investigate the small-molecule capturing capability of the MNPR, we prepared different $p$-ATP concentrations for use in quantitative analysis (Fig. 4). In this experiment, we compared the normalized resonant frequency shift between a commercial resonator (grey bar) and MNPR (blue bar) with respect to $p$-ATP concentrations from $10 \mu \mathrm{M}$ to $1 \mathrm{nM}$. First, in order to characterize the capturing sensitivity, we normalized the resonant frequency of the MNPR due to the diverse initial resonance of each individual cantilever. The normalized resonant frequency is described as $\omega_{\mathrm{m}}=\left(\omega_{\mathrm{c}}-\omega_{\mathrm{n}}\right) / \omega_{\mathrm{b}} \times 100$, where $\omega_{\mathrm{c}}$ and $\omega_{\mathrm{n}}$ are the resonant frequencies of the capturing of a small molecule using MNPR and the Au nanoporous state, respectively, and $\omega_{\mathrm{b}}$ is the resonant frequency of the bare Au state resonator. In Fig. 4, the normalized resonant frequency shift of the MNPR shows high sensitivity to the p-ATP compared with the bare $\mathrm{Au}$ resonator using well-known gold-sulphur chemistry. Here the limit of detection (LOD) of MNPR is $1 \mathrm{nM}$ $\left(0.110 \pm 0.091, \omega_{\mathrm{m}} \pm\right.$ s.d. $)$, which shows a $\sim 10,000$ times improvement over the LOD of the commercial resonator $(10 \mu \mathrm{M}$, $0.167 \pm 0.03)$. As the molecular weight of $p$-ATP $(\mathrm{MW}=125.19$ $\mathrm{Da}$ ) is extremely small, the normal resonator is not able to track the concentration (Supplementary Fig. 4). For the statistical validity and reproducibility of preparation process, resonant frequencies of five different MNPRs were measured for each concentration ${ }^{14,43}$. The $0 \mathrm{M}$ measurement indicates the control experiment using only ethanol solution $(0 \mathrm{M}, 0.010 \pm 0.023$ for MNPR and $0.008 \pm 0.021$ for the commercial resonator, Supplementary Fig. 5). The proposed sensing platform has better sensitivity than our previous studies, which used a commercial method that was only able to amplify the mass ${ }^{9,10,14}$. Moreover, our MNPR has a possibility for increasing the sensitivity using a

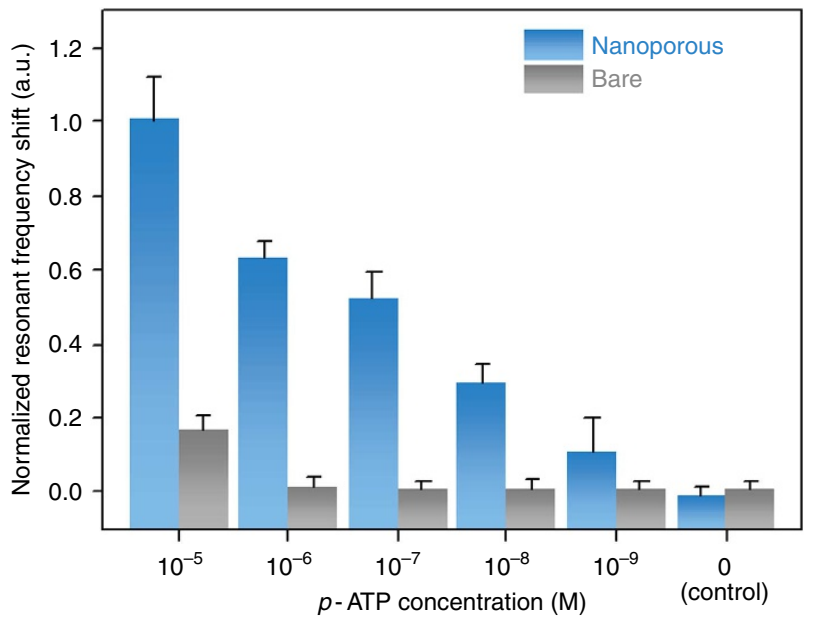

Figure 4 | Resonant sensing using MNPR. Normalized resonant frequency shift of MNPR and bare resonator with respect to $p$-ATP concentration. The blue bars and grey bars indicate the normalized resonant frequency shift of MNPR and bare resonator, respectively. $\mathrm{OM}$ indicates the control experiment. For the statistical data, resonant frequencies of five different MNPR were measured for each concentration. Each error bar indicates the s.d.

properly designed mass amplifier ${ }^{14,43}$. In this experiment, the detection limit of $p$-ATP increase to $10 \mathrm{pM}$ using sandwich assay amplifier (Supplementary Fig. 6). Here, the PEG $(5 \mathrm{kDa})$ molecules acted as a mass 9 .

SERS sensing. In the viewpoint of a resonator, though MNPR have a magnificent sensitivity, MNPR shows constraints such as target-molecule discrimination due to the characteristic of resonance-based sensing method. However, MNPR does allow for 
a

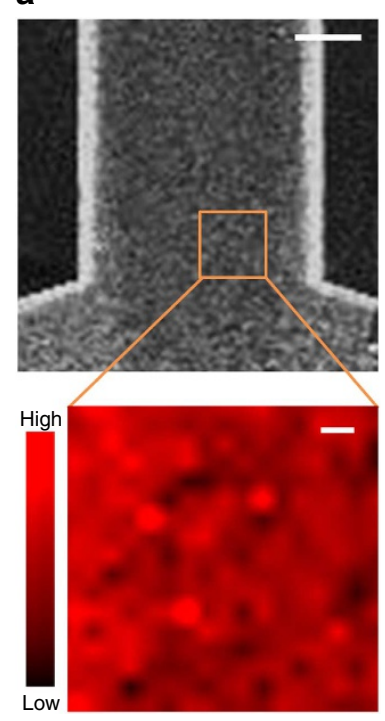

b

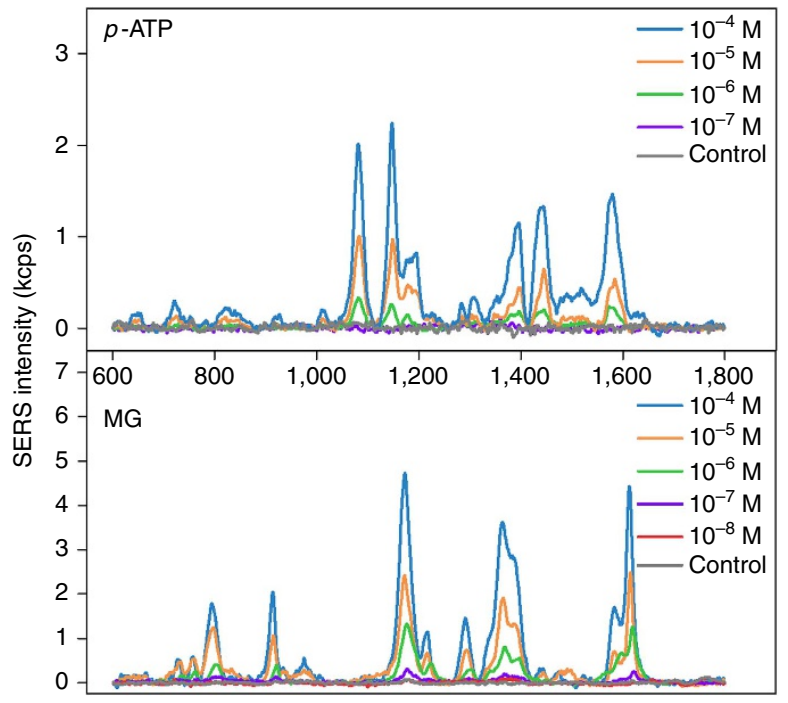

Raman shift $\left(\mathrm{cm}^{-1}\right)$ c
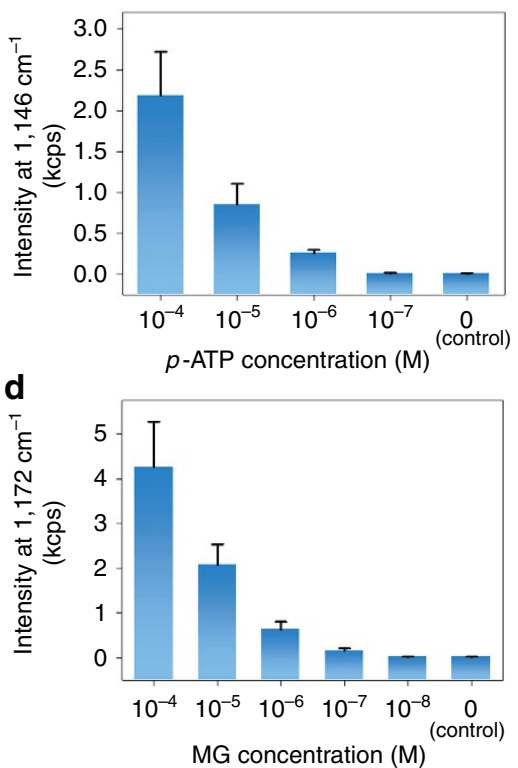

Figure 5 | SERS sensing using MNPR. (a) SEM and confocal Raman-mapped image of MNPR using $p$-ATP molecules. Raman signal was imaged between 1,140 and $1,145 \mathrm{~cm}^{-1}$ (top scale bar is $10 \mu \mathrm{m}$ and bottom scale bar is $1 \mu \mathrm{m}$ ). (b) SERS spectra of MNPR with different concentrations of $p$-ATP and MG. (c) SERS intensity $\left(1,146 \mathrm{~cm}^{-1}\right)$ of MNPR against $\mathrm{p}-\mathrm{ATP}$ concentration. For the statistical data, five different MNPR were measured for each concentration. Each error bar indicates the s.d. (d) SERS intensity $\left(1,172 \mathrm{~cm}^{-1}\right.$ ) of MNPR against MG concentration. The laser $(\lambda=633 \mathrm{~nm})$ on the MNPR was $500 \mu \mathrm{W}$ and the integration time was $10 \mathrm{~s}$. For the statistical data, five different MNPR were measured for each concentration. Each error bar indicates the s.d.

another method of discrimination target molecules. For the proof of concept, we have taken into account the SERS response using MNPR because Raman scattering gives molecule-specific information (Fig. 5) ${ }^{16}$. Prior to the discrimination of captured small molecules using MNPR, we investigated the feasibility of utilizing MNPR as a SERS substrate using confocal Raman mapping on MNPR after small-molecule capture ( $p$-ATP, $100 \mu \mathrm{M}$ ) (Fig. 5a). We mapped the intensity of the SERS signal from a $10 \times 10 \mu \mathrm{m}$ square at the centre-neck site of the resonator. It was shown that the significant SERS signal was fairly uniformly observed in comparison with several reports (note the brightness of the red colour at bottom image $)^{17,40}$. Therefore, we measured the SERS signal of the MNPR after the capture of two different small molecules for various solution concentrations: $p$-ATP and MG (Fig. 5b). The upper graph in Fig. 5b is the SERS signal of MNPR after the capture of $p$-ATP. The characteristic Raman scattering peak at $1,079 \mathrm{~cm}^{-1}\left(a_{1}\right.$ band of $p$-ATP) and three additional peaks at $1,145,1,383$ and $1,436 \mathrm{~cm}^{-1}\left(\mathrm{~b}_{2}\right.$ band of DMAB, oxidatively transformed from $p$-ATP) were observed ${ }^{28,41,42}$. In contrast, after the capture of MG, the characteristic Raman scattering peaks at $1,180,1,370$ and $1,618 \mathrm{~cm}^{-1}$ were observed ${ }^{44}$. The characteristic Raman scattering peaks of the two captured small molecules are different. Furthermore, we expect that molecular-specific discrimination of any captured small molecule is possible using the SERS measurement of the MNPR. We also depicted peak SERS intensity of each molecule with different concentrations for quantitative analysis (Fig. $5 c, d$ ). The LOD for SERS discrimination of $p$-ATP (Fig. $5 c$ ) is $1 \mu \mathrm{M}$ and for MG (Fig. 5d) the LOD is $100 \mathrm{nM}$. The LOD of MG is 10 -fold greater than that of $p$-ATP because the electronic transition of MG is in resonance with the frequency of the incident laser light ${ }^{45}$.

\section{Discussion}

In summary, we have reported a novel approach based on MNPR that has not only shown high sensitivity but also excellent target molecule discrimination. The optimal fabrication process of MNPR was determined by carrying out a surface area analysis using Raman mapped images provided by tmAFM and confocal Raman microscopy. The LOD of MNPR is $1 \mathrm{nM}$ of which sensitivity is 10,000 times higher than commercial resonator to small-molecule detection. It was also shown that MNPR was able to discriminate the binding molecules $1 \mu \mathrm{M}$ for $p$-ATP and $100 \mathrm{nM}$ MG molecules using SERS response. It is noted that until recently these methods have not been available, which can provide multimodal high-sensitive detection and discriminational information of binding molecules. It is due to the limitation of conventional detection methods. However, as described in this work, MNPR is able to endow an insight into fusion detection method, which accumulates the advantages of label-free sensing methods from merely simple and swift fabrication process. Furthermore, because of AFM-Raman technique ${ }^{46}$ the MNPR has possibility for commercializing toward various target molecules. In the long run, it is implied that MNPR may enable not only basic label-free sensitive sensor platform capable of revealing an insight into the efficacy of general biological, chemical, environmental and blood sensor ${ }^{47,48}$ but also the fiducial point for multimodal detection.

\section{Methods}

MNPR fabrication. For the fabrication of MNPR, Au/Ag alloy was electrochemically deposited on the Au surface of micro a cantilever and Ag was selectively etched into the surface. The PPP-NCHAu (NANOSENSORS, Switzerland) cantilever was used as a resonator with dimensions of $30 \times 4 \times 125 \mu \mathrm{m}^{3}$ (width $\times$ thickness $\times$ length) and a force constant of $\sim 42 \mathrm{~N} \mathrm{~m}^{-1}$. A metallic layer of (Au) was coated on both sides of the resonator that improved the contact area. The resonant frequency of the resonator was $\sim 240 \mathrm{kHz}$, which was an appropriate frequency for cantilever sensors in air ${ }^{9,14}$. However, for quantitative analysis we normalized the resonant frequency of the resonator due to different initial values of the various cantilevers ${ }^{14}$. Prior to electrochemical deposition, the Au-coated micro resonators were sequentially cleaned with acetone, ethanol and deionized water. In order to determine the exposed area for Au/Ag alloy deposition, the non-exposed area was passivated using lacquer. Electrochemical experiments were performed 
using a potentiostat (CompactStat, Ivium) that was controlled by a computer A conventional three-electrode system was used for this experiment with a platinum ( $\mathrm{Pt}$ ) wire counter electrode, an $\mathrm{Ag} / \mathrm{AgCl}(1 \mathrm{M} \mathrm{KCl})$ reference electrode and a working electrode. $\mathrm{Au} / \mathrm{Ag}$ alloy was electrochemically deposited by using a mixture of gold and silver-plating stock solutions. A gold-plating stock solution was prepared by dissolving $20 \mathrm{mM}$ gold cyanide in an aqueous media and the $\mathrm{pH}$ was controlled using $\mathrm{Na}_{2} \mathrm{CO}_{3}$ and the silver-plating stock solution was prepared by dissolving $20 \mathrm{mM}$ silver cyanide rather than gold cyanide. A mixture of goldplating stock solution and silver-plating stock solution was prepared in various

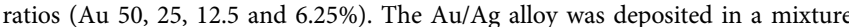
solution at $-0.8 \mathrm{~V}$ versus $\mathrm{Ag} / \mathrm{AgCl}$ for $180 \mathrm{~s}$. The reaction was performed in a temperature-controlled vessel at $10^{\circ} \mathrm{C}$. Thereafter, selective etching of $\mathrm{Ag}$ was performed by immersion of as-prepared $\mathrm{Au} / \mathrm{Ag}$ alloy-deposited micro-cantilever in nitric acid $\left(\mathrm{HNO}_{3}\right)$ for $2 \mathrm{~s}$. After selective etching, the MNPR was washed with ethanol and triple-distilled water (Millipore, Bedford, MA, $\mathrm{pH}$ 7.5) several times until it was clean. After the washing process was complete, the MNPR was dried under vacuum for $6 \mathrm{~h}$ at room temperature.

Synthesis of PEG conjugated $\boldsymbol{p}$-ATP. Synthesis of PEG-conjugated $p$-ATP was carried out by well-known amine-NHS carboxylate-based method. In detail, p-ATP and monomethoxy-NHS-activated PEG (mPEG-NHS; MW: 5k) were allowed to react in chloroform for $24 \mathrm{~h}$ at room temperature. Then, the resultatnt products were dialysed (MWCO: $1 \mathrm{k}$ ) to exlude unreacted $p$-ATP (Supplementary Fig. 6).

TmAFM and SEM images and analysis. TmAFM measurements were performed using an Innova (Bruker Corp., CA, USA) with a NanoDrive controller (Bruker Corp) in air at ambient temperature and pressure. The clear images were obtained using a closed-loop scanner, which allows precise, reproducible images of each state. For an accurate imaging, the ultra-sharp tip which name is SSS-NCHR (NANOSENSORS, Switzerland) cantilever tip was used for recording the twodimensional (2D) and 3D images. The tip radius of this cantilever was $\sim 2 \mathrm{~nm}$ and the resonant frequency was $\sim 300 \mathrm{kHz}$. All images were $3 \times 3 \mu \mathrm{m}$ in size with a scanning frequency of at $0.8 \mathrm{~Hz}$. Two-dimensional levelling processes were performed for all images using SPM Lab Analysis software V 7.00 (Bruker Corp.). The surface area analysis was performed using Nanoscope analysis software V 1.20 (Bruker Corp.). The morphology of the MNPRs was observed by FE-SEM (JSM6500F, JEOL).

p-ATP detection comparison and control experiment. For the resonant frequency shift of both MNPR and the bare resonator due to the $p$-ATP (SigmaAldrich Chemicals) absorption, AFM was used to measure (Innova with Nanodrive controller). The $p$-ATP had an amine group $\left(\mathrm{NH}_{2}\right)$ and thiol group $(\mathrm{SH})$ on both ends. In order to facilitate $p$-ATP adsorption on the MNPR and the bare resonator surface, both resonators were immersed in the ethanol-based p-ATP buffer solution for $1 \mathrm{~h}$. The $p$-ATP molecules were attached on both the Au surface (bare resonator and the MNPR) using well-known gold-sulpgur chemistry. In order to confirm the possibility of physical absorption on the surface, we prepared ethanol solution without $p$-ATP (Supplementary Fig. 5). We observed that the resonant frequency shifts of the bare resonator and the MNPR did not occur in the control experiment (Fig. 4, 0 M). This resonant frequency shift shows the reliability results comparing with our previous work ${ }^{9,14,43}$. The statistical data were normalized for the resonant frequency shift of five different resonators and data were obtained at each concentration.

Raman spectra imaging and analysis. Raman spectroscopy measurements were performed using confocal Raman microscopy (LabRAM ARAMIS, Horiba). A $\times 100$ microscope objective (Nikon, NA $=0.95)$ and a He-Ne laser $(\lambda=633 \mathrm{~nm})$ were used to obtain the high-quality images. The laser power on the sample was $\sim 50 \mu \mathrm{W}$, and the integration time was $10 \mathrm{~s}$. A silicon wafer with a Raman band at $520 \mathrm{~cm}^{-1}$ was used to calibrate the spectrometer. For confocal Raman imaging spectroscopy, the laser power on the sample was $\sim 0.5 \mathrm{~mW}$, the integration time was $1 \mathrm{~s}$ per spectrum and the scanning step was $0.5 \mu \mathrm{m}$. LabSpec 5 software (Horiba) was used for spectral and image processing and analysis.

EDS measurement. FE-SEM and EDS were performed with a FE-SEM (JSM$7100 \mathrm{~F}$, JEOL) equipped with an energy-dispersive X-ray analyser at an accelerating voltage of $20 \mathrm{kV}$ (Supplementary Fig. 2)

Electrochemical estimation of surface area. Electrochemical experiments for estimation of surface area were also performed using a potentiostat (CompactStat, Ivium). A conventional three-electrode system was used for this experiment with a platinum $(\mathrm{Pt})$ wire counter electrode, an $\mathrm{Ag} / \mathrm{AgCl}(1 \mathrm{M} \mathrm{KCl})$ reference electrode and a working electrode (Bare, Au 50\%, Au 25\%, Au 12.5\% and Au 6.25\%). Cyclic voltammetry was used to estimate the electrochemically active surface area. Cyclic voltammetries were performed in $0.5 \mathrm{M}$ sulphuric acid in the potential range between $-0.2 \mathrm{~V}$ (initial value) and $1.8 \mathrm{~V}$ (versus $\mathrm{Ag} / \mathrm{AgCl}$ ) at a scan rate of
$10 \mathrm{mV} \mathrm{s}^{-1}$. For each working electrode, 20 cycles were performed in order to achieve reliable measurements. For surface-area esimation, gold oxide reduction peak $(0.6$ to $1.2 \mathrm{~V})$ was integrated.

\section{References}

1. Johnson, B. N. \& Mutharasan, R. Biosensing using dynamic-mode cantilever sensors: a review. Biosens. Bioelectron. 32, 1-18 (2012).

2. Waggoner, P. S. \& Craighead, H. G. Micro- and nanomechanical sensors for environmental, chemical, and biological detection. Lab Chip 7, 1238-1255 (2007).

3. Krug, H. F. \& Wick, P. Nanotoxicology: An Interdisciplinary Challenge. Angew Chem. Int. Ed. 50, 1260-1278 (2011).

4. Hempen, C. \& Karst, U. Labeling strategies for bioassays. Anal. Bioanal. Chem 384, 572-583 (2006).

5. Orosco, M. M., Pacholski, C. \& Sailor, M. J. Real-time monitoring of enzyme activity in a mesoporous silicon double layer. Nat. Nanotechnol. 4, 255-258 (2009).

6. Arlett, J. L., Myers, E. B. \& Roukes, M. L. Comparative advantages of mechanical biosensors. Nat. Nanotechnol. 6, 203-215 (2011).

7. Goeders, K. M., Colton, J. S. \& Bottomley, L. A. Microcantilevers: sensing chemical interactions via mechanical motion. Chem. Rev. 108, 522-542 (2008).

8. Li, M., Tang, H. X. \& Roukes, M. L. Ultra-sensitive NEMS-based cantilevers for sensing, scanned probe and very high-frequency applications. Nat. Nanotechnol. 2, 114-120 (2007).

9. Kwon, T. et al. Nanomechanical in situ monitoring of proteolysis of peptide by cathepsin B. PLoS One 4, e6248 (2009).

10. Lee, G. et al. Real-time quantitative monitoring of specific peptide cleavage by a proteinase for cancer diagnosis. Angew. Chem. Int. Ed. 51, 5837-5841 (2012)

11. Kwon, T. et al. Micromechanical observation of the kinetics of biomolecular interactions. Appl. Phys. Lett. 93, 173901 (2008).

12. Tae Yun, K. et al. In situ real-time monitoring of biomolecular interactions based on resonating microcantilevers immersed in a viscous fluid. Appl. Phys Lett. 90, 223903 (2007).

13. Burg, T. P. et al. Weighing of biomolecules, single cells and single nanoparticles in fluid. Nature 446, 1066-1069 (2007).

14. Park, J., Choi, W., Jang, K. \& Na, S. High-sensitivity detection of silver ions using oligonucleotide-immobilized oscillator. Biosens. Bioelectron. 41, 471-476 (2013).

15. Moskovits, M. Imaging: Spot the hotspot. Nature 469, 307-308 (2011).

16. Willets, K. A. \& Van Duyne, R. P. Localized surface plasmon resonance spectroscopy and sensing. Annu. Rev. Phys. Chem. 58, 267-297 (2007).

17. Bang, D. et al. One-step electrochemical fabrication of vertically self-organized silver nanograss. J. Mater. Chem. A 1, 4851-4857 (2013).

18. Im, H., Bantz, K. C., Lindquist, N. C., Haynes, C. L. \& Oh, S. H. Vertically oriented sub-10-nm plasmonic nanogap arrays. Nano Lett. 10, 2231-2236 (2010).

19. Clark, A. W. \& Cooper, J. M. Nanogap ring antennae as plasmonically coupled SERRS substrates. Small 7, 119-125 (2011)

20. Lo, K.-H., Chen, M.-C., Ho, R.-M. \& Sung, H.-W. Pore-filling nanoporous templates from degradable block copolymers for nanoscale drug delivery. ACS Nano 3, 2660-2666 (2009).

21. Jackson, E. A. \& Hillmyer, M. A. Nanoporous membranes derived from block copolymers: from drug delivery to water filtration. ACS Nano 4, 3548-3553 (2010).

22. Cohen-Tanugi, D. \& Grossman, J. C. Water desalination across nanoporous graphene. Nano Lett. 12, 3602-3608 (2012).

23. Ge, M., Rong, J., Fang, X. \& Zhou, C. Porous doped silicon nanowires for lithium ion battery anode with long cycle life. Nano Lett. 12, 2318-2323 (2012).

24. Backmann, N. et al. A label-free immunosensor array using single-chain antibody fragments. Proc. Natl Acad. Sci. USA 102, 14587-14592 (2005).

25. Zhang, L. et al. Nanoporous gold based optical sensor for sub-ppt detection of mercury ions. ACS Nano 7, 4595-4600 (2013).

26. Hotta, K., Yamaguchi, A. \& Teramae, N. Nanoporous waveguide sensor with optimized nanoarchitectures for highly sensitive label-free biosensing. ACS Nano 6, 1541-1547 (2012).

27. Dutta, P. et al. Enantioselective sensors based on antibody-mediated nanomechanics. Anal. Chem. 75, 2342-2348 (2003).

28. Huang, Y.-F. et al. Surface-enhanced Raman spectroscopic study of p-aminothiophenol. Phys. Chem. Chem. Phys. 14, 8485-8497 (2012).

29. Zong, S., Wang, Z., Yang, J. \& Cui, Y. Intracellular pH sensing using p-aminothiophenol functionalized gold nanorods with low cytotoxicity. Anal. Chem. 83, 4178-4183 (2011).

30. Li, M. et al. Detection of adenosine triphosphate with an aptamer biosensor based on surface-enhanced Raman scattering. Anal. Chem. 84, 2837-2842 (2012).

31. Lohmüller, T. et al. Single molecule tracking on supported membranes with arrays of optical nanoantennas. Nano Lett. 12, 1717-1721 (2012). 
32. Lee, D., Shin, N., Lee, K.-H. \& Jeon, S. Microcantilevers with nanowells as moisture sensors. Sens. Actuators B 137, 561-565 (2009).

33. Braun, T. et al. Micromechanical mass sensors for biomolecular detection in a physiological environment. Phys. Rev. E 72, 031907 (2005).

34. Dasary, S. S. R., Singh, A. K., Senapati, D., Yu, H. \& Ray, P. C. Gold nanoparticle based label-free SERS probe for ultrasensitive and selective detection of trinitrotoluene. J. Am. Chem. Soc. 131, 13806-13812 (2009).

35. Lim, D.-K. et al. Highly uniform and reproducible surface-enhanced Raman scattering from DNA-tailorable nanoparticles with 1-nm interior gap. Nat. Nanotech. 6, 452-460 (2011).

36. Ogi, H., Yanagida, T., Hirao, M. \& Nishiyama, M. Replacement-free massamplified sandwich assay with $180-\mathrm{MHz}$ electrodeless quartz-crystal microbalance biosensor. Biosens. Bioelectron. 26, 4819-4822 (2011).

37. Li, Y., Koshizaki, N., Wang, H. \& Shimizu, Y. Untraditional approach to complex hierarchical periodic arrays with trinary stepwise architectures of micro-, submicro-, and nanosized structures based on binary colloidal crystals and their fine structure enhanced properties. ACS Nano 5, 9403-9412 (2011).

38. Dasary, S. S., Singh, A. K., Senapati, D., Yu, H. \& Ray, P. C. Gold nanoparticle based label-free SERS probe for ultrasensitive and selective detection of trinitrotoluene. J. Am. Chem. Soc. 131, 13806-13812 (2009).

39. Yan, B. et al. Engineered SERS substrates with multiscale signal enhancement: nanoparticle cluster arrays. ACS Nano 3, 1190-1202 (2009).

40. Osinkina, L., Lohmüller, T., Jäckel, F. \& Feldmann, J. Synthesis of gold nanostar arrays as reliable, large-scale, homogeneous substrates for surface-enhanced raman scattering imaging and spectroscopy. J. Phys. Chem. C 117, 22198-22202 (2013).

41. Huang, Y.-F. et al. When the signal is not from the original molecule to be detected: chemical transformation of para-aminothiophenol on Ag during the SERS measurement. J. Am. Chem. Soc. 132, 9244-9246 (2010).

42. Liu, G.-K. et al. Laser-induced formation of metal - molecule - metal junctions between Au nanoparticles as probed by surface-enhanced raman spectroscopy. J. Phys. Chem. C 112, 6499-6508 (2008).

43. Jang, K. et al. Highly sensitive detection of self-aggregated single-walled carbon nanotubes using a DNA-immobilized resonator. Chem. Commun. 49, 8635-8637 (2013)

44. Efremov, E. V., Ariese, F. \& Gooijer, C. Achievements in resonance Raman spectroscopy review of a technique with a distinct analytical chemistry potential. Anal. Chim. Acta 606, 119-134 (2008).
45. Mahajan, S., Baumberg, J. J., Russell, A. E. \& Bartlett, P. N. Reproducible SERRS from structured gold surfaces. Phys. Chem. Chem. Phys. 9, 6016-6020 (2007).

46. Łojewska, J., Knapik, A., Kołodziej, A. \& Jodłowski, P. Far field combined AFM and micro-Raman imaging for characterisation of surface of structured catalysts: example of Pd doped $\mathrm{CoO}_{\mathrm{x}}$ catalysts on precalcined kanthal steel. Top. Catal. 56, 1088-1095 (2013).

47. Qian, L. H., Yan, X. Q., Fujita, T., Inoue, A. \& Chen, M. W. Surface enhanced Raman scattering of nanoporous gold: Smaller pore sizes stronger enhancements. Appl. Phys. Lett. 90, 153120 (2007)

48. Liu, H. et al. Single molecule detection from a large-scale SERS-active Au79Ag21 substrate. Sci. Rep. 1, 112 (2011).

\section{Acknowledgements}

This research was supported by the National Research Foundation of Korea (NRF) under grant nos NRF-2013-044213, ERC-2007-0056094 and NRF-2013058102, which is funded by the Ministry of Science, ICT \& Future Planning. Further, this work was supported by BioNano Health-Guard Research Center funded by the Ministry of Science, ICT \& Future Planning (MSIP) of Korea as Global Frontier Project (grant no. H-GUARD_2013M3A6B2078946).

\section{Author contributions}

J.P., D.B., S.H. and S.N. conceived the project; J.P., D.B. and K.J. designed and performed the experiment; K.J. and E.K. analysed the data; S.H. and S.N. wrote the paper.

\section{Additional information}

Supplementary Information accompanies this paper at http://www.nature.com/ naturecommunications

Competing financial interests: The authors declare no competing financial interests

Reprints and permission information is available online at http://npg.nature.com/ reprintsandpermissions.

How to cite this article: Park, J. et al. Multimodal label-free detection and discrimination for small molecules using a nanoporous resonator. Nat. Commun. 5:3456 doi: $10.1038 /$ ncomms4456 (2014). 